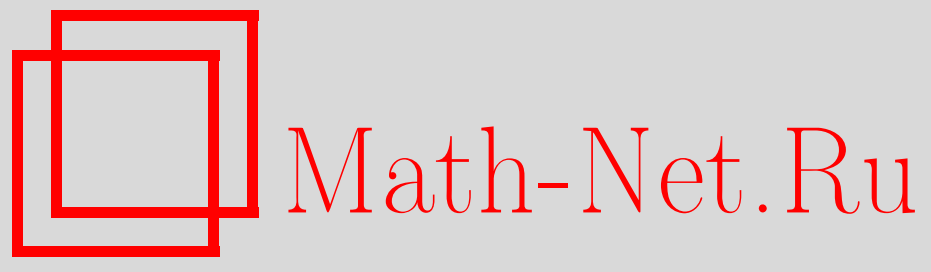

Э. А. Стороженко, Л. Г. Коваленко, Неравенство для дробных интегралов комплексных полиномов в $L_{0}, M a$ тем. заметки, 2014, том 96, выпуск 4, 633-636

DOI: https://doi.org/10.4213/mzm10532

Использование Общероссийского математического портала Math-Net.Ru подразумевает, что вы прочитали и согласны с пользовательским соглашением http://www . mathnet.ru/rus/agreement

Параметры загрузки:

IP: 34.229 .108 .108

26 апреля 2023 г., 13:41:42

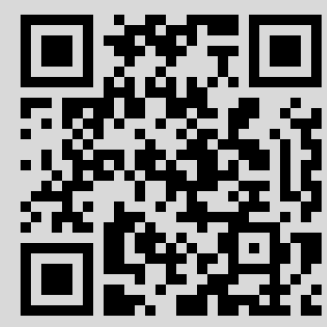




\section{Неравенство для дробных интегралов комплексных полиномов в $L_{0}$}

\section{Э. А. Стороженко, Л. Г. Коваленко}

Пусть $P_{n}(z)=\sum_{k=0}^{n} c_{k} z^{k}-$ алгебраический полином степени $n$ с комплексными коэффициентами. Функционалы $\|\cdot\|_{p}$ на единичной окружности $|z|=1$ при $0 \leqslant p \leqslant \infty$, как обычно, определяются равенствами

$$
\begin{gathered}
\left\|P_{n}\right\|_{\infty}=\max \left|P_{n}(z)\right|, \quad\left\|P_{n}\right\|_{p}=\left(\frac{1}{2 \pi} \int_{0}^{2 \pi}\left|P_{n}\left(e^{i \varphi}\right)\right|^{p} d \varphi\right)^{1 / p}, \\
\left\|P_{n}\right\|_{0}=\lim _{p \rightarrow 0}\left\|P_{n}\right\|_{p}=\exp \left(\frac{1}{2 \pi} \int_{0}^{2 \pi}\left|P_{n}\left(e^{i \varphi}\right)\right|^{p} d \varphi\right)^{1 / p} .
\end{gathered}
$$

Следуя Малеру [1], квазинорму $\left\|P_{n}\right\|_{0}$ будем называть мерой полинома $P_{n}(z)$ и обозначать $M\left(P_{n}\right)$.

Усилиями многих математиков доказано неравенство, позволяющее оценивать нормы или квазинормы производных полиномов $P_{n}(z)$ через нормы или квазинормы самих $P_{n}(z)$ :

$$
\left\|P_{n}^{\prime}\right\|_{p} \leqslant n\left\|P_{n}\right\|_{p}, \quad 0 \leqslant p \leqslant \infty
$$

(с подробной информацией можно ознакомиться в статье Арестова [2]).

Задачу об оценке снизу $\left\|P_{n}^{\prime}\right\|_{p}$ посредством $\left\|P_{n}\right\|_{p}$ можно сформулировать как аналог неравенства (1) для неопределенного интеграла

$$
I P_{n}(z)=\int_{0}^{z} P_{n}(t) d t=z \sum_{k=0}^{n} c_{k} \frac{z^{k}}{k+1},
$$

если $P_{n}(z)$ заменить на $P_{n}^{\prime}(z)$.

В самом деле, $I P_{n}^{\prime}(z)=P_{n}(z)-P_{n}(0)$, и тогда оценка $\left\|I P_{n}^{\prime}\right\|_{p}$ сверху через $\left\|P_{n}^{\prime}\right\|_{p}$ дает ответ на поставленную задачу:

$$
\left\|I P_{n}^{\prime}\right\|_{p}=\left\|P_{n}(z)-P_{n}(0)\right\|_{p} \leqslant A_{n, p}\left\|P_{n}^{\prime}\right\|_{p}, \quad 0 \leqslant p \leqslant \infty .
$$

Заменяя в (2) $P_{n}^{\prime}$ на $P_{n}$, получаем неравенство типа Бернштейна для неопределенного интеграла

$$
\left\|I P_{n}\right\|_{p} \leqslant A_{n, p}\left\|P_{n}\right\|_{p}, \quad 0 \leqslant p \leqslant \infty,
$$

которое часто называют неравенством типа Фавара.

За последние двадцать лет вырос интерес к дробному интегродифференцированию, о чем свидетельствуют многочисленные публикации по этой тематике. В настоящей статье рассматриваются дробные интегралы порядка $0<\alpha<1$ и доказывается аналог неравенства $\left(2^{\prime}\right)$ для мер полиномов.

Вполне логично для полинома $P_{n}$ определить дробный интеграл порядка $0<\alpha<1$ равенством

$$
I^{\alpha} P_{n}(z)=\sum_{k=1}^{n} c_{k} \frac{z^{k}}{(k+1)^{\alpha}} .
$$

Такое определение встречается, например, в работах Флетт (см. [3], [4]) для аналитических функций $f(z)$ внутри единичного круга в связи с изучением потенциалов Бесселя и Рисса.

DOI: $10.4213 / \operatorname{mzm} 10532$ 


\section{Справедлива}

Теорема. Для любого полинома $P_{n}(z)$ имеет место неравенство

$$
M\left(I^{\alpha} P_{n}\right) \leqslant A_{\alpha, n} M\left(P_{n}\right), \quad 0<\alpha<1,
$$

где

$$
A_{\alpha, n}<\frac{A_{\alpha}}{n^{\alpha}}(1,4)^{n}, \quad A_{\alpha}<\frac{2 \alpha+1}{\alpha \Gamma(\alpha)} .
$$

Порядок роста коэффициента $A_{\alpha, n}$ является точным в том смысле, что он совпадает с порядком точного значения коэффичиента в неравенстве для неопределенного интеграла, т.е. при $\alpha=1$.

Для сравнения мер полиномов $I^{\alpha} P_{n}$ и $P_{n}$ удобно воспользоваться одним результатом де Брюйна и Спрингера [5] (см. также Арестов [6]), справедливым для композиции полиномов по Сегё (см. [7; V, с. 75]). Напомним, что композицией полиномов

$$
Q_{n}(z)=\sum_{k=0}^{n} C_{n}^{k} b_{k} z^{k} \quad \text { и } \quad R_{n}(z)=\sum_{k=0}^{n} C_{n}^{k} d_{k} z^{k}
$$

называют полином

$$
Q_{n} \otimes R_{n}(z)=\sum_{k=0}^{n} C_{n}^{k} b_{k} d_{k} z^{k}
$$

и тогда

$$
M\left(Q_{n} \otimes R_{n}\right) \leqslant M\left(Q_{n}\right) M\left(R_{n}\right) .
$$

Поэтому данный полином $P_{n}$ запишем в виде $P_{n}(z)=\sum_{k=0}^{n} C_{n}^{k} a_{k} z^{k}$ и, соответственно,

$$
I^{\alpha} P_{n}(z)=\sum_{k=0}^{n} C_{n}^{k} a_{k} \frac{z^{k}}{(k+1)^{\alpha}} .
$$

Следовательно, $I^{\alpha} P_{n}(z)=P_{n} \otimes I^{\alpha}(1+z)^{n}$. Согласно неравенству (4) доказательство теоремы сводится к вычислению меры полинома $I^{\alpha}(1+z)^{n}$ :

$$
A_{\alpha, n}=M\left(I^{\alpha}(1+z)^{n}\right) .
$$

ДокАзАтЕльство. Из определения гамма-функции Эйлера $Г(\alpha)$ следует равенство

$$
\frac{1}{(k+1)^{\alpha}}=\frac{1}{\Gamma(\alpha)} \int_{0}^{\infty} x^{\alpha-1} e^{-(k+1) x} d x, \quad \alpha>0, \quad k>-1 .
$$

Тогда полином $I^{\alpha}(1+z)^{n}$ представим в интегральной форме

$$
I^{\alpha}(1+z)^{n}=\sum_{k=0}^{n} C_{n}^{k} \frac{z^{k}}{(k+1)^{\alpha}}=\frac{1}{\Gamma(\alpha)} \int_{0}^{\infty}\left(1+e^{-x} z\right)^{n} e^{-x} x^{\alpha-1} d x .
$$

Оценим его модуль на единичной окружности $z=e^{i \varphi}$, разбивая при этом интеграл $\int_{0}^{\infty}$ на два: $I_{1}=\int_{0}^{1 / n}$ и $I_{2}=\int_{1 / n}^{\infty}$.

Так как

$$
\left|1+e^{-x} e^{-i \varphi}\right|^{2}=1+2 e^{-x} \cos \varphi+e^{-2 x} \leqslant 1+e^{-x}(2 \cos \varphi+1) \leqslant 2(1+\cos \varphi),
$$

то

$$
\left|I_{1}\right| \leqslant\left(2 \cos \frac{\varphi}{2}\right)^{n} \int_{0}^{1 / n} x^{\alpha-1} d x \leqslant \frac{(2 \cos \varphi / 2)^{n}}{n^{\alpha}}
$$


и

$$
\begin{aligned}
\left|I_{2}\right| & \leqslant \int_{1 / n}^{\infty}\left|1+e^{-x} e^{-i \varphi}\right|^{n} e^{-x} x^{\alpha-1} d x \leqslant \frac{1}{n^{\alpha-1}} \int_{0}^{\infty}\left(1+e^{-x}(2 \cos \varphi+1)\right)^{n / 2} e^{-x} d x \\
& =\frac{1}{n^{\alpha-1}} \cdot \frac{2}{n+2} \cdot \frac{[1+(2 \cos \varphi+1)]^{n / 2+1}-1}{2 \cos \varphi+1} \\
& \leqslant \frac{2}{n^{\alpha}} \cdot \frac{1}{|2 \cos \varphi+1|} \cdot \begin{cases}\left(2 \cos \frac{\varphi}{2}\right)^{n+2}, & \varphi \in\left(0, \frac{2 \pi}{3}\right), \\
1, & \varphi \in\left(\frac{2 \pi}{3}, \pi\right) .\end{cases}
\end{aligned}
$$

Следовательно,

$$
\begin{aligned}
\left|I_{1}+I_{2}\right| \leqslant \frac{(2 \cos \varphi / 2)^{n}}{\alpha n^{\alpha}}+\frac{2}{n^{\alpha}} \cdot \begin{cases}\frac{(2 \cos \varphi / 2)^{n+2}}{(2 \cos \varphi+1)} \\
\frac{1}{|2 \cos \varphi+1|}\end{cases} \\
\leqslant \frac{2 \alpha+1}{\alpha} \cdot \frac{1}{n^{\alpha}} \cdot \begin{cases}\frac{(2 \cos \varphi / 2)^{n+2}}{(2 \cos \varphi+1)}, & \varphi \in\left(0, \frac{2 \pi}{3}\right), \\
\frac{1}{|2 \cos \varphi+1|}, & \varphi \in\left(\frac{2 \pi}{3}, \pi\right)\end{cases}
\end{aligned}
$$

(при $\varphi \in(0,2 \pi / 3)$ использовалось неравенство $2 \cos \varphi+1 \leqslant(2 \cos \varphi / 2)^{2}$, а при $\varphi \in(2 \pi / 3, \pi)$ учитывалось, что $\left.(2 \cos \varphi / 2)^{n}<1\right)$.

Теперь по определению меры (см. (5))

$$
\begin{aligned}
A_{\alpha, n} & \leqslant \frac{2 \alpha+1}{\alpha \Gamma(\alpha)} \cdot \frac{1}{n^{\alpha}} \cdot \exp \left(\frac{1}{\pi} \int_{0}^{2 \pi / 3} \ln \frac{(2 \cos (\varphi / 2))^{n+2}}{2 \cos \varphi+1} d \varphi-\frac{1}{\pi} \int_{2 \pi / 3}^{\pi} \ln |2 \cos \varphi+1| d \varphi\right) \\
& =\frac{2 \alpha+1}{\alpha \Gamma(\alpha)} \cdot \frac{1}{n^{\alpha}} \cdot \exp \left(\frac{n+2}{\pi} \int_{0}^{2 \pi / 3} \ln \left(2 \cos \frac{\varphi}{2}\right) d \varphi-\frac{1}{\pi} \int_{0}^{\pi} \ln |2 \cos \varphi+1| d \varphi\right) .
\end{aligned}
$$

Первый интеграл выразим посредством функции Лобачевского $L(x)=-\int_{0}^{x} \ln \cos x d x$ и воспользуемся ее табличным значением (см. [8]):

$$
\frac{1}{\pi} \int_{0}^{2 \pi / 3} \ln \left(2 \cos \frac{\varphi}{2}\right) d \varphi=\frac{2}{3} \ln 2-\frac{2}{\pi} L\left(\frac{\pi}{3}\right) \approx 0.323 .
$$

Второй интеграл равен нулю:

$$
\frac{1}{\pi} \int_{0}^{\pi} \ln |2 \cos \varphi+1| d \varphi=2 \ln 2+\frac{4}{\pi} \int_{0}^{\pi / 2} \ln \cos t d t=0 .
$$

Из (6)-(8) следует (3).

Теперь относительно порядка роста коэффициента $A_{\alpha, n}$. В статье [9] в связи с одной задачей Малера находится

$$
M\left(I(1+z)^{n}\right)=\frac{1}{n} \Pi_{n / 6 \leqslant k \leqslant 5 n / 6} 2 \sin \frac{\pi k}{n},
$$

что по порядку составляет

$$
\frac{(1,4)^{n}}{n}, \quad n \rightarrow \infty .
$$

Теорема доказана. 


\section{СПИСОК ЦИТИРОВАННОЙ ЛИТЕРАТУРЫ}

[1] K. Mahler, Proc. Roy. Soc. London Ser. A, 264 (1961), 145-154. [2] B. B. Арестов, Матем. заметки, 48:4 (1990), 7-18. [3] T. M. Flett, Pacif. J. Math., 25:3 (1968), 463-494. [4] T. M. Flett, "Temperatures, Bessel potentials and Lipschitz Spaies", Proc. London Math. Soc. (3), 22:3 (1971), 385-451. [5] N. G. de Bruijn, T. A. Springer, Indag. Math., 9 (1947), 406-414. [6] В. В. Арестов, Изв. АН СССР. Сер. матем., 45:1 (1981), 3-22. [7] Г. Полиа, Г. Сёге, Задачи и теоремы из анализа. Ч. 2. Теория функиий. Распределение нулей. Полиномы. Определители. Теория чисел, Наука, М., 1978. [8] И. С. Грандштейн, И. М. Рыжик, Таблиць интегралов, сумм и произведений, ГИТТЛ, М., 1964. [9] Э. А. Стороженко, Матем. сб., 187:5 (1996), 111-120.

\section{Э. А. Стороженко}

Одесский национальный университет им. И. И. Мечникова, Украина

\section{Л. Г. Коваленко}

Одесский национальный университет им. И. И. Мечникова, Украина

E-mail: baier@ukr.net
Поступило 21.01.2014 